\title{
US summit: neonatal spine - how to do it
}

\author{
Harriet J. Paltiel
}

(C) Springer-Verlag 2011

Sonography of the spine is performed as a screening examination in neonates with cutaneous lesions associated with spinal cord abnormalities, such as deep sacral dimples, dorsal midline masses, hemangiomas, skin tags, hairy tufts, skin discoloration and sinus tracts; congenital anomalies with a high incidence of occult spinal dysraphism, including imperforate anus, caudal regression syndrome, cloacal malformation and cloacal exstrophy; vertebral body anomalies; and tumors located near the vertebral canal such as neuroblastoma, sacrococcygeal teratoma and presacral masses in patients with Currarino triad. Additional indications include assessment of hemorrhage following birth injury or lumbar puncture, and in the detection of epidural abscesses.

Infants are usually examined in the prone position. A small bolster or pillow placed under the pelvis accentuates the lumbar lordosis and thereby aids in the sonographic delineation of the lumbosacral junction. If prone positioning is not possible, the study can also be performed with the infant lying on his or her side. A neonate who is fed immediately prior to or during the examination will usually lie quietly as scanning is performed.

The neonatal spinal cord can be assessed with US due to a lack of ossification of the predominantly cartilaginous posterior arches of the spine. As the posterior spinal elements progressively ossify, the quality of the US images declines and, in most cases, is inadequate by 6 months of age. In

Disclaimer Dr. Paltiel has no financial interests, investigational or offlabel uses to disclose.

H. J. Paltiel $(\bowtie)$

Children's Hospital Boston,

Boston, MA 02115, USA

e-mail: harriet.paltiel@childrens.harvard.edu patients with spinal dysraphism, however, the persisting acoustic window permits US scanning at any age. Most studies are limited to an evaluation of the lumbosacral spine, as, for example, in the investigation of a sacral dimple or to determine the presence of a hematoma after a traumatic or unsuccessful lumbar puncture. However, in selected patients, an examination of the entire spinal canal is performed, from the craniocervical junction to the coccyx. Scanning is generally accomplished with high-frequency linear-array transducers with images obtained in transverse and longitudinal planes. The craniocervical junction is best imaged with a smaller footprint sector transducer to permit optimal imaging through the foramen magnum.

The normal neonatal spinal cord appears as a tubular, hypoechoic structure with hyperechoic walls. The central canal is hyperechoic, and the surrounding subarachnoid space is anechoic. The vertebral bodies located anterior to the spinal canal appear as discrete, echogenic structures. In the axial plane, the spinal cord is round or oval in shape. Echogenic dentate ligaments can be seen extending laterally from the spinal cord to attach to the arachnoid and dura mater. The cord gradually tapers at the level of the first or second lumbar vertebral body to form the conus medullaris. The fibrous filum terminale arises from the tip of the conus medullaris and extends into the sacral canal. The filum appears echogenic and is surrounded by the echogenic nerve roots of the cauda equina. A cystic structure is occasionally noted at the tip of the conus medullaris. This terminal ventricle, or filar cyst, is a normal developmental variant.

The level of the conus medullaris must be determined in every patient. It usually lies above the level of the L2 vertebral body, although it can extend as low as the upper third of the L3 vertebral body. A low-lying cord can result from tethering and requires further evaluation with MRI. Determination of vertebral level can be accomplished in a 
variety of ways: finding the $12^{\text {th }}$ rib and counting downward, counting upward from the lumbosacral junction or counting upward from the tip of the coccyx. When none of these approaches is satisfactory, the US transducer can be used to locate the tip of the conus and a radiopaque marker placed on the skin followed by a radiograph to assess vertebral level.

The dorsoventral position of the cord should be assessed. It is usually located a third to halfway between the anterior and posterior walls of the spinal canal. If it lies more posteriorly, tethering should be suspected.

The cord and nerve roots should always be evaluated for normal pulsatile movement. Tethering leads to an absence of pulsatility. The thickness of the filum terminale should measure $2 \mathrm{~mm}$ or less. A thickened filum might be caused by the presence of a fibrolipoma or can be a manifestation of the "tight filum terminale syndrome"; both entities can lead to tethering of the spinal cord. Vertebral anomalies such as block vertebrae and dysraphism should be documented.

In conclusion, screening sonography of the neonatal spine permits a timely assessment of the spinal canal and a distinction between patients requiring urgent intervention and those in whom additional imaging studies such as MRI can be delayed or avoided. 